\title{
Mission Roles: Status, Issues, and Recommendations for the Planetary Science and Astrobiology Decadal Committee Consideration
}

Corresponding Author: Julie Castillo-Rogez, Jet Propulsion Laboratory, California Institute of Technology, Julie.C.Castillo@jpl.nasa.gov

\section{Co-Authors:}

Christina Richey, Jet Propulsion Laboratory, California Institute of Technology, christina.r.richey@jpl.nasa.gov

Robert Pappalardo, Jet Propulsion Laboratory, California Institute of Technology, robert.pappalardo@jpl.nasa.gov

Shawn Brooks, Jet Propulsion Laboratory, California Institute of Technology, shawn.m.brooks@jpl.nasa.gov

Ingrid J. Daubar, Brown University, ingrid daubar@brown.edu

Elizabeth Turtle, Johns Hopkins Applied Physics Lab, Elizabeth.Turtle@jhuapl.edu

Kate Craft, Johns Hopkins Applied Physics Lab, Kate.Craft@jhuapl.edu

Candice Hansen, Planetary Science Institute, cjhansen@psi.edu

Carolyn Ernst, Johns Hopkins Applied Physics Lab, Carolyn.Ernst@jhuapl.edu

Diana Blaney, Jet Propulsion Laboratory, California Institute of Technology,

Diana.L.Blaney@jpl.nasa.gov

Julie Rathbun, Planetary Science Institute, rathbun@psi.edu

Acknowledgements: A portion of the work described was carried out at the Jet Propulsion Laboratory, California Institute of Technology, under a contract with the National Aeronautics and Space Administration (80NM0018D0004). 


\section{Scope}

This white paper addresses issues identified with current mission roles for scientists at the home institution of the mission, such as Project Scientist (PS), Deputy PS, Investigation Scientist (IS), and Project Staff Scientist ${ }^{1}$ (PSS), and the paper offers recommendations for the Decadal Survey Committee's and NASA's consideration. A separate white paper by Prockter et al. covers the participating scientist and guest investigator roles.

Both approaches, internal selection and competed selection, are important in distinct ways towards both broadening participation and diversity as well as creating opportunities for leadership experience for future generations, but each is a unique subset. This white paper addresses the different pathways that exist for scientists to gain mission leadership experience and recommends that those pathways be fully utilized in uniform, consistent ways to allow for the greatest opportunity for the community.

\section{Key Findings}

- Investigation scientists and project staff scientists are critical to the success of planetary missions.

- The strength that the dPS, IS, or PSS brings is the interdisciplinary part of the job: the knowledge of science, engineering, and operations. The Instrument PIs depend on ISs to stay aware of project impacts on instrument development and triage technical issues that may impact science. Similarly the PS depends on the dPSs and PSSs to interface with the spacecraft and systems engineering teams as well as the scientists in the PSG to suss out issues that may affect the science of the mission.

$\circ$ US ISs support non-US instrument teams, which are common on NASA missions and generally require a higher level of support than most US instruments to address differences in mission implementation with NASA and in some cases to represent the instruments during the US workday when large time differences are involved.

0 The IS is employed by the project and is the primary liaison between the investigation team PI and the mission's Project Scientist. The basic responsibility of an IS is to know what is going on in the project every day and what might affect their investigation and serve as a liaison. The PI should have an IS that they trust to speak on behalf of the instrument team and to know when to

\footnotetext{
${ }^{1}$ Project Staff Scientist (PSS) is part of the project science team and is a role that supports PSG functionality and interactions of PS, and dPSs with Investigation PIs and Engineering.
} 
elevate an issue to the PI. Very often the IS works directly with instrument and project engineers and science teams to resolve small issues to prevent them from becoming critical issues.

- The IS and PSS roles evolve over the life cycle of a mission. The need doesn't go away but specific activities change as missions progress through design and development, and then to operations.

- One of the important roles the dPSs and PSSs fulfill is to support PSG functionality between teams and between Project Science and the Investigation teams.

- All science team members, in particular ISs and PSSs, are doing a service to the community in their mission role with delayed gratification and delivery of data to the community; remaining active in science (at minimum, attending conferences and keeping up with recent research activities) is an essential part of being an effective IS and PSS, and is particularly critical for early career scientists. However, not all ISs and PSSs receive formal funding and support for research activities in addition to their mission responsibilities.

- Investigation Scientist (IS) and Project Staff Scientist (PSS) roles offer opportunities to learn about and participate in missions, especially during the development phase; this kind of role is one of the most effective ways to see all the types of challenges that occur during mission phases A-D.

- Avenues for scientists to gain experience during mission development phases are scarce. However, this experience is crucial to effective future participation and leadership in planetary science missions.

- IS and PSS roles provide essential support to the missions themselves, as well as investments in the community's future.

- Although the Discovery/New Frontiers AO does encourage the training of the next generation of mission leaders, they leave it to the mission projects to decide on the inclusion of these roles and the extent of their functions: "Training the next generation of mission leaders is a priority for NASA, and science missions under the Discovery and New Frontiers programs present tremendous opportunities for such training. Proposers are encouraged to include career development opportunities in science, engineering, and management areas of their proposed mission. For example, generous use of deputies for team members allow individuals to gain experience and expertise, preparing them to assume lead roles in future missions." 
- These roles lack a consistent formal definition and their implementation depends on each institution and project. Missions often employ ISs differently. Juno does not formally have ISs, even though some scientists actually perform IS functions.

- The lack of guidelines from NASA to mission implementing centers results in different appreciation of these roles from project to project, which can have a negative impact on people's careers, undercut the ability to obtain optimal science, and reduce the training opportunity for the next generation, which hurts the current mission and missions to come. A recent example is the unexpected removal of several of the Mars 2020 Perseverance ISs immediately after launch, a large fraction of whom were early career scientists and representatives from minorities. Such a change not only impacts the ISs themselves, but also the mission and mission team in that it reduces communication among the team and continuity between the development phases and upcoming operations.

- Not all PSs or ISs receive research support on top of mission-related activities, and even when they do, they do not necessarily have time set aside from their mission responsibilities to be able to do research. On Cassini, some ISs were supported only 0.1 FTE during the extended mission. Europa Clipper is taking a different approach by providing $0.5 \mathrm{FTE}$, with $20-50 \%$ of their time designated for research.

\section{Recommendations}

- We recommend that NASA should study how these roles are a critical career path for underrepresented groups.

- Important early career opportunity, being aware of EDI issues is very important in nurturing and developing career paths.

- Data should be collected to evaluate how many PSs had prior experience as ISs. In particular, there is some anecdotal evidence that women PIs and PSs were more likely to have previously been an IS.

- There need to be specific definitions of the Project Scientist, Deputy Project Scientist, Investigation/Instrument Scientist, and Project Staff Scientist roles, for each mission, written by the leadership of that mission, and a recognition of the true value of investing in and integrating these key roles in the missions.

- IS opportunities should be offered as part of mission announcements of opportunity and commitments should be made during Phase A (or earlier), i.e., not be a floating role that may or may not be fulfilled depending on mission management's whim. 
- Minimum standards need to be clearly established for these roles in a way that ensures respect by science teams and management.

- Active participation in research is an essential part of these roles. It should be encouraged and explicitly stated as a job responsibility. Maintaining a presence in the research community allows ISs and PSSs to better serve their instrument teams and missions by being up-to-date with science issues that may be of interest to their teams and allowing them to communicate those issues within the project.

- The Europa Clipper Project in particular has laid out specific definitions for the Deputy-PS, PSS, and IS jobs as well as ground rules that can serve as a reference for future projects.

- NASA should provide guidelines for all missions to avoid issues like the recent occurrence on Mars 2020 Perseverance ISs, so that this critical role does not find itself on the chopping block overnight.

- Besides these avenues for training Project Scientists and future mission and instrument PIs, additional opportunities are also needed to broaden mission leadership opportunities. One such recent example was the PI Launchpad Workshop hosted by NASA. Another is the Planetary Science Summer School hosted by JPL.

- Extended missions offer an opportunity for influx of new personnel and this should be pursued - see "Extended Missions in Planetary Science: Impacts to Science and the Workforce", by I. J. Daubar and R. A. Beyer et al.

- Other programs (like SIMPLEX) provide another venue for forming mission leaders but comes with so little funding that PIs do not get in-depth experience with managing a substantial science team (basically <0.2-0.3 FTE total for WBS 4 throughout the mission, leaving little funding available during cruise) 